\title{
Non-Gaussian simulation using Hermite polynomial expansion: convergences and algorithms
}

\author{
Bénédicte Puig a,b, Fabrice Poirion ${ }^{\mathrm{a}, *}$, Christian Soize $^{\mathrm{c}}$ \\ a ONERA/MS DDSS, Centre de Chatillon Leclerc, BP 7229 avenue de la Division, F 92322 Chatillon cedex, France \\ ${ }^{\mathrm{b}}$ Pierre et Marie Curie University, Paris 6, France \\ ${ }^{\mathrm{c}}$ University of Marne la Vallée, Marne la Vallée, France
}

\begin{abstract}
Mathematical justifications are given for a Monte Carlo simulation technique based on memoryless transformations of Gaussian processes. Different types of convergences are given for the approaching sequence. Moreover an original numerical method is proposed in order to solve the functional equation yielding the underlying Gaussian process autocorrelation function. (C) 2002 Elsevier Science Ltd. All rights reserved.

Keywords: Monte Carlo simulation; Non Gaussian process; Hermite polynomial expansion
\end{abstract}

\section{Introduction}

Due to the formidable progress of computer technology, Monte Carlo simulation (MCS) methods are leaving their benchmark method status to become fully effective methods which are more and more integrated in industrial codes. Consequently, industry is relying more heavily on MCS methods for decreasing the design and construction costs of their products, performing for instance damage and fatigue optimization. In that context, they need to use stochastic loads which match real-life loads which, as many examples have shown, are typically non-Gaussian rather than Gaussian processes.

Various methods have been proposed for generating simulated paths of non-Gaussian processes [8 10,15,16,19 21,27]. The main conceptual difficulty lies in the characterization of the process: unlike Gaussian processes which are determined solely through their first- and secondorder probabilistic characteristics, one must know the entire family of joint distributions $\left\{\mathscr{L}\left(X_{t_{1}}, \ldots, X_{t_{n}}\right), n \geq 1, t_{i} \in \mathbb{R}\right\}$. Of course, such a data is never available (at least for real-life processes), and one has to deal with a truncated characterization of the non-Gaussian process. The reasonable minimum amount of information used to 'approach' the real behavior of the non-Gaussian process should at least include the onedimension marginal probability distribution and the correlation function. However, even the one-dimension marginal distribution is not available and one has to deal instead with a given number of statistical moments often.

Another essential aspect of MCS methods which has to be considered in order to ensure the soundness of the method, as it is done for Gaussian simulation [18], is the convergence behavior of approximation.

The goal of this paper is to propose a general method to generate simulated paths of non-Gaussian homogeneous random fields, based, as it is done for instance in Refs. $[3,20,21]$, on a Hermite polynomial expansion, given the spectral measure of the random field and either the onedimension marginal distribution or a fixed number of statistical moments. Different types of convergence will be given for the approximating sequence. It will be shown how the problem of determining the autocorrelation function of the underlying Gaussian process can be approached by an optimization problem. Two formulations will be given, whether the underlying Gaussian process is generated using a spectral approach method or a Markovian representation method. Finally, results of applications including the various aspects of the method will be given.

\section{Method description}

Let $(\Omega, \mathscr{A}, \mathbb{P})$ be a probability space. For any $x \in \mathbb{R}$, Hermite polynomials are defined by:

$H_{0}(x) \quad 1, \quad H_{n}(x) \quad(1)^{n} \mathrm{e}^{x^{2} / 2} \frac{\mathrm{d}^{n}}{\mathrm{~d} x^{n}} \mathrm{e}^{\left(x^{2} / 2\right),} \quad n \in \mathbb{N}^{*}$. 


\subsection{Data}

It is aimed to simulate the paths of a strictly stationary non-Gaussian process $\left(Y_{t}, t \in \mathbb{R}^{+}\right)$whose statistical description is reduced either to a finite number of moments or to its one-dimension marginal distribution. Two sets of data will therefore be considered:

Case 1.

(i) Let $\mu_{1}, \mu_{2}, \ldots, \mu_{N}(N>1)$ be real numbers which are statistical moments of a random variable. We can assume in the following that $\mu_{1} \quad 0, \mu_{2} \quad 1$.

(ii) Let $R: \mathbb{R} \rightarrow \mathbb{R}$ be a function in $L^{2}(\mathbb{R}, \mathrm{d} x)$ such that $R(0) \quad 1, R$ is nonnegative definite.

\section{Case 2.}

(i) A cumulative distribution function $F_{Y}$ of a random variable $Y$ is given, with $\mathbb{E}\left(Y^{2}\right) \quad 1$.

(ii) Let $R: \mathbb{R} \rightarrow \mathbb{R}$ be a function in $L^{2}(\mathbb{R}, \mathrm{d} x)$ such that $R(0) \quad 1, R$ is nonnegative definite.

Gaussian simulation methods are well known $[4,7,11,12,18,22,25]$ and very simple to utilize. That explains why many methods use nonlinear transformations of Gaussian process in order to simulate non-Gaussian ones. Moreover, the family $\left((\sqrt{n !}){ }^{1} H_{n}\right)_{n \in \mathbb{N}}$ is an orthonormal base of $L^{2}\left(\mathbb{R},\left(\left(\mathrm{e}^{\left(x^{2} / 2\right)} / \sqrt{2 \pi}\right)\right) \mathrm{d} x\right)$. It is then natural to construct a strictly stationary process $\left(Y_{t}, t \in \mathbb{R}^{+}\right)$defined by the relation

$Y_{t} \quad \sum_{n=1}^{\infty} f_{n} H_{n}\left(G_{t}\right)$,

where $H_{n}$ is the Hermite polynomial of degree $n,\left(G_{t}, t \in\right.$ $\mathbb{R}^{+}$) is a standard stationary Gaussian process (i.e. for every fixed $t, G_{t}$ has a zero-mean, unit variance Gaussian distribution), such that either $\mathbb{E}\left(Y_{t}^{n}\right) \quad \mu_{n} \forall n \in\{1, \ldots, N\}$, (case 1) or for every fixed $t$, the random variables $Y_{t}$ and $Y$ have the same distribution (case 2), and such that the autocorrelation function $R_{Y}$ of $\left(Y_{t}, t \in \mathbb{R}^{+}\right)$is close to $R$ in the Hilbert space $L^{2}(\mathbb{R}, \mathrm{d} x)$.

Whether the first statistical moments or the marginal distribution are given, different assumptions must be verified. However, at the end, it is the same general method which is used: find a function $f$ and a Gaussian process $G_{t}$ such that

$Y_{t} \equiv f\left(G_{t}\right)$.

Eq. (3) means that the two processes have the same given statistical data.

\subsection{Necessary condition and sufficient condition for $\left(\mu_{1}, \ldots, \mu_{N}\right)$}

The considered simulation method for the case 1 requires the determination of a continuous (necessary condition to construct the cumulative distribution function) distribu- tion having the first $N$ moments equal to $\left(\mu_{1}, \ldots, \mu_{N}\right)$. Let $\left(\mu_{n}\right)_{n \in \mathbb{N}^{*}}$ be a sequence of real numbers such that $\tilde{\mu}_{n}:=(n+1) \mu_{n}$, for all $n \in\{1, \ldots, N\}$. The continuous distribution will be constructed as the product of a discrete distribution with moments $\tilde{\mu}_{n}$ by an independent uniform distribution. Not all finite sequence of real numbers can be defined as the moments of a distribution, a condition must be fulfilled, which is recalled below. In fact, the above product gives a random variable with an unimodal distribution.

\subsubsection{Necessary condition}

Theorem 2.1 [23]. If there exists a random variable $\tilde{Y}$ such that $\mathbb{E}\left(\tilde{Y}^{k}\right) \quad \tilde{\mu}_{k}$ for all $k \in \mathbb{N}^{*}$, then for all $k \in \mathbb{N}^{*}$

$D_{k}:=\operatorname{det}\left(\begin{array}{cccc}1 & \tilde{\mu}_{1} & \cdots & \tilde{\mu}_{k} \\ \tilde{\mu}_{1} & \tilde{\mu}_{2} & \cdots & \tilde{\mu}_{k+1} \\ \vdots & \vdots & \ddots & \vdots \\ \tilde{\mu}_{k} & \tilde{\mu}_{k+1} & \cdots & \tilde{\mu}_{2 k}\end{array}\right) \geq 0$

\subsubsection{Sufficient condition}

Theorem 2.2 [23]. Using the notations of Theorem 2.1, if there exists $K$ such that

$\begin{cases}\forall k \leq K & D_{k}>0, \\ \forall k>K & D_{k} \quad 0,\end{cases}$

then there exists a discrete random variable $\tilde{Y}$ (unique in distribution) such that its distribution has $(K+1)$ atoms and $\mathbb{E}\left(\tilde{Y}^{k}\right) \quad \tilde{\mu}_{k}$ for all $k \in \mathbb{N}^{*}$.

Remark 2.3. There exist various techniques to generate such a random variable, see for instance Devroye's book [5]: the atoms of $\tilde{Y}$ are then determined as well as the attached probabilities. Finally, the statistical moments of the random variable $Y \quad U \tilde{Y}$, where $U$ has an uniform probability distribution over $[0,1]$ and which is independent of $\tilde{Y}$, are $\left(\mu_{1}, \ldots, \mu_{N}\right)$. The probability distribution of $Y$ is absolutely continuous with respect to the Lebesgue measure $\left(\exists p_{Y} \in L^{1}(\mathbb{R}, d x), F_{Y}(x) \quad \int^{x}{ }_{\infty} p_{Y}(x) \mathrm{d} x\right)$. Its cumulative distribution function can be obtained analytically.

\subsection{Utilization of Hermite polynomials}

The first step is to identify the nonlinear function $f$ appearing in the memoryless transformation (3). This function is constructed using the cumulative distribution functions of the given non-Gaussian process $Y_{t}$ and of a standard normal random variable. In what follows, the function $F_{Y}$ denotes either the cumulative distribution 
function of the random variable $Y$ described in Section 2.1 for case 1 , or the data itself for case 2 .

The inverse of the cumulative distribution function $F_{Y}$ is defined by

$F_{Y}{ }^{1}(y) \quad \inf \left\{x \in \mathbb{R} / F_{Y}(x) \geq y\right\}$

(where $\inf (\emptyset) \quad+\infty)$. The cumulative distribution function of $F_{Y}{ }^{1}(U)$, where $U$ is a random variable with an uniform probability distribution over $[0,1]$, is $F_{Y}$. If $G$ is the standard normal random variable $\mathscr{N}(0,1)$ and $F_{G}$ its cumulative distribution function, $F_{G}(G)$ has an uniform distribution over $[0,1]$. So the cumulative distribution function of the random variable $F_{Y}{ }^{1} \circ F_{G}(G)$ is $F_{Y}$. Thus the following hypothesis is considered:

$F_{Y}{ }^{1} \circ F_{G} \in L^{2}\left(\mathbb{R}, \frac{e^{\left(x^{2} / 2\right)}}{\sqrt{2 \pi}} \mathrm{d} x\right)$.

If this assumption is true, then the function $F_{Y}{ }^{1} \circ F_{G}$ can be projected on the base $\left(\left(\sqrt{n !}{ }^{1}\right) H_{n}\right)_{n \in \mathbb{N}}$ : there exists a real sequence $\left(f_{n}\right)_{n}$ such that

$\forall x \in \mathbb{R}, \quad F_{Y}{ }^{1} \circ F_{G}(x) \quad \sum_{n=0}^{\infty} f_{n} H_{n}(x)$,

where

$f_{n} \quad(n !) \int_{\mathbb{R}} F_{Y}{ }^{1} \circ F_{G}(x) H_{n}(x) \frac{e^{\left(x^{2} / 2\right)}}{\sqrt{2 \pi}} \mathrm{d} x$,

the series being convergent in $L^{2}\left(\mathbb{R},\left(\mathrm{e}^{\left(x^{2} / 2\right)} / \sqrt{2 \pi}\right) \mathrm{d} x\right)$.

Proposition 2.4. Let $\left(G_{t}, t \in \mathbb{R}^{+}\right)$be a standard stationary Gaussian process and $R_{G}$ its autocorrelation function. Then the process $\left(Y_{t}, t \in \mathbb{R}^{+}\right)$defined by

$Y_{t} \quad F_{Y}^{1} \circ F_{G}\left(G_{t}\right)$

is strictly stationary, and

$\mathbb{E}\left(Y_{t}^{n}\right) \quad \mu_{n}, \quad \forall n \in\{1, \ldots, N\}$.

Proof. As it was remarked above, $Y_{t} \quad F_{Y}{ }^{1} \circ F_{G}\left(G_{t}\right)$ has $F_{Y}$ for cumulative distribution function and has then $\left(\mu_{1}, \ldots, \mu_{N}\right)$ for first moments. The second step is to identify the underlying Gaussian process $G_{t}$ used in relation (3). Its autocorrelation function is solution of a functional equation:

$R_{Y}(t) \quad \sum_{n=1}^{\infty}(n !) f_{n}^{2} R_{G}(t)^{n}$.

This is the difficult point in this method because it is not guaranteed that Eq. (12) has a solution, and even if a solution exists, it has to be a nonnegative definite function. Two numerical methods based on an optimization technique will be further given in order to construct an autocorrelation function 'approaching' a solution of Eq. (12).

\section{Convergence results}

Let $\left(Y_{t}^{M}\right)_{M}$ be the sequence defined by

$Y_{t}^{M} \quad \sum_{n=1}^{M} f_{n} H_{n}\left(G_{t}\right)$.

Our goal is to study the convergence of the sequence $\left(Y_{t}^{M}\right)_{M}$ towards $Y_{t}$ as $M \rightarrow \infty$. Various convergence results of the truncated sum sequence will be given.

\subsection{Mean-square convergence}

Proposition 3.1. For any fixed the sequence $\left(Y_{t}^{M}\right)_{M \in \mathbb{N}^{*}}$ converges uniformly in $t$ towards $Y_{t}$ in the space $L^{2}(\Omega, A, \mathbb{P})$.

Proof. Owing to the transport of measure, the coefficients $\left(f_{n}\right)$ are given by

$f_{n} \quad(n !){ }^{1} \mathbb{E}\left(Y_{t} H_{n}\left(G_{t}\right)\right)$,

(n!) ${ }^{1} \mathbb{E}\left(F_{Y}^{1} \circ F_{G}\left(G_{t}\right) H_{n}\left(G_{t}\right)\right)$

$\left(\begin{array}{ll}f_{0} & 0 .\end{array}\right)$

Since $G_{t}$ is stationary, $f_{n}$ does not depend on $t$. Therefore for any fixed $t$, we have

$Y_{t} \quad F_{Y}{ }^{1} \circ F_{G}\left(G_{t}\right)$,

$Y_{t} \quad \sum_{=1}^{\infty} f_{n} H_{n}\left(G_{t}\right)$

in $L^{2}(\Omega, \mathscr{A}, \mathbb{P})$.

We will now prove that the autocorrelation function of the truncated sum converges towards the target autocorrelation function.

Proposition 3.2. Let $R_{M}$ denotes the autocorrelation function of $\left(Y_{t}^{M}, t \in \mathbb{R}^{+}\right)$and $R_{Y}$ the autocorrelation function of $\left(Y_{t}, t \in \mathbb{R}\right)$

$R_{M}(t) \underset{M \rightarrow \infty}{\rightarrow} R_{Y}(t), \quad \forall t \in \mathbb{R}$.

Lemma 3.3 (Mehler Formula Formula [3]). Let $\left(G_{t}, t \in\right.$ $\mathbb{R}^{+}$) a zero-mean Gaussian process such that $\mathbb{E}\left[G_{t}^{2}\right] \quad 1$ for all $t \in \mathbb{R}^{+}$and let $R_{G}(t, s)$ be its autocorrelation function. Then

$\mathbb{E}\left[H_{n}\left(G_{t}\right) H_{m}\left(G_{s}\right)\right] \quad n !\left(R_{G}(t, s)\right)^{n} \delta_{n m}$

(where $\delta$ denotes the Kronecker symbol).

Using Mehler's formula:

$R_{Y}(t \quad s) \quad \mathbb{E}\left(Y_{t} Y_{s}\right)$, 


$$
\begin{aligned}
& R_{Y}(t \quad s) \quad \mathbb{E}\left(F_{Y}^{1} \circ F_{G}\left(G_{t}\right) F_{Y}{ }^{1} \circ F_{G}\left(G_{s}\right)\right), \\
& R_{Y}(t \quad s) \quad \sum_{m, n} f_{m} f_{n} \mathbb{E}\left(H_{n}\left(G_{t}\right) H_{m}\left(G_{t}\right)\right), \\
& R_{Y}(t \quad s) \quad \sum_{n}(n !) f_{n}^{2} R_{G}(t \quad s)^{n}, \\
& R_{Y}(t \quad s) \quad \lim _{M \rightarrow \infty} \sum_{n=1}^{M}(n !) f_{n}^{2} R_{G}(t \quad s)^{n},
\end{aligned}
$$

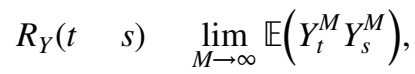

$$
\begin{aligned}
& R_{Y}(t \quad s) \quad \lim _{M \rightarrow \infty} R_{M}(t \quad s) .
\end{aligned}
$$

\subsection{Error evaluation}

By assuming some decreasing conditions for the coefficients $f_{n}$, an evaluation of the error due to the truncation can be obtained.

Proposition 3.4. If there exists a constant $C>0$ such that for any $n$

$f_{n}^{2} \leq \frac{C}{(n !)^{2}}$,

then for any fixed $t$

$\left|R_{Y}(t) \quad R_{M}(t)\right| \leq \frac{C}{M M !}\left|R_{G}(t)\right|^{M+1}$

$\left|R_{Y}(t) \quad R_{M}(t)\right| \leq \frac{C}{M M !}$.

Remark 3.5. If the function $f:=F_{Y}{ }^{1} \circ F_{G}$ is a $C^{\infty}$ function which $n$th derivatives are uniformly bounded in $n$ by a positive constant $K>0$, then the assumption is checked. As a matter of fact, using integration by parts, we have

$f_{n} \quad(n !){ }^{1} \mathbb{E}\left(f^{(n)}\left(G_{t}\right)\right)$.

Thus

$f_{n}^{2} \leq \frac{K^{2}}{n !^{2}}$.

Proof. The autocorrelation functions are given, respectively, by

$R_{Y}(t) \quad \sum_{n=1}^{\infty}(n !) f_{n}^{2} R_{G}(t)^{n}$

and

$R_{M}(t) \quad \sum_{n=1}^{M}(n !) f_{n}^{2} R_{G}(t)^{n}$
On the other hand, using Cauchy Schwarz inequality and using the fact that, for any $t$, the $G_{t}$ distribution is $\mathscr{N}(0,1)$

$\left|R_{G}(t)\right| \quad \mathbb{E}\left(G_{0} G_{t}\right)$,

$\left|R_{G}(t)\right| \leq \mathbb{E}\left(G_{0}^{2}\right)^{1 / 2} \mathbb{E}\left(G_{t}^{2}\right)^{1 / 2}$,

$\left|R_{G}(t)\right| \leq 1$.

Therefore

$\begin{array}{ll}\left|R_{Y}(t) \quad R_{M}(t)\right| & \left|\sum_{n=M+1}^{\infty}(n !) f_{n}^{2} R_{G}(t)^{n}\right|, \\ \left|R_{Y}(t) \quad R_{M}(t)\right| \leq \sum_{n=M+1}^{\infty}(n !) f_{n}^{2}\left|R_{G}(t)\right|^{M+1},\end{array}$

$\left|R_{Y}(t) \quad R_{M}(t)\right| \leq C \sum_{n=M+1}^{\infty}(n !){ }^{1}\left|R_{G}(t)\right|^{M+1}$,

$\left|R_{Y}(t) \quad R_{M}(t)\right| \leq \frac{C}{M M !}\left|R_{G}(t)\right|^{M+1}$

$\left|R_{Y}(t) \quad R_{M}(t)\right| \leq \frac{C}{M M !}$.

The last but one inequality is of course finer than the last. But the quantity $\left|R_{G}(t)\right|$ is unknown.

Corollary 3.6. Under assumption of Remark 3.5 and for any $t$

$E\left(\left(\begin{array}{ll}Y_{t} & Y_{t}^{M}\end{array}\right)^{2}\right) \leq \frac{C}{M M !}$

Proof.

$E\left(\left(\begin{array}{ll}Y_{t} & Y_{t}^{M}\end{array}\right)^{2}\right) \quad \mathbb{E}\left(Y_{t}^{2}\right) \quad 2 \mathbb{E}\left(Y_{t} Y_{t}^{M}\right)+\mathbb{E}\left(\left(Y_{t}^{M}\right)^{2}\right)$,

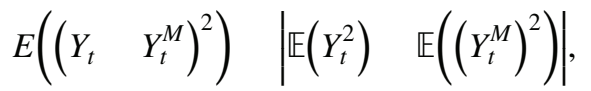

$E\left(\left(\begin{array}{ll}Y_{t} & Y_{t}^{M}\end{array}\right)^{2}\right) \quad\left|R_{Y}(0) \quad R_{M}(0)\right|$

The proof is concluded using Remark 3.5 with $t \quad 0$.

\subsection{Almost sure convergence}

Assuming now a stronger condition on the sequence $\left(f_{n}\right)$, almost sure convergence can be proved.

Proposition 3.7. If

$\sum_{n=1}^{\infty}(\ln (n))^{2} f_{n}^{2}(n !)<\infty$, 
then for any fixed the sequence $\left(Y_{t}^{M}\right)_{M \in \mathbb{N}^{*}}$ converges a.s. towards $Y_{t}$.

Lemma 3.8 [13]. Let $\left(Z_{n}\right)_{n}$ be a sequence of second-order random variables which are orthogonal. If

$\sum_{n \in \mathbb{N}^{*}}(\ln \mathrm{n})^{2} \mathbb{E}\left(Z_{n}^{2}\right)<\infty$,

then the sum $\sum_{n \in \mathbb{N}^{*}} Z_{n}$ converges almost surely.

$\left(H_{n}\left(G_{t}\right)_{n \in \mathbb{N}^{*}}\right.$ is a sequence of random variables which are orthogonal in $L^{2}(\Omega, \mathscr{A}, \mathbb{P})$, so Proposition 3.7 is proved by using Lemma 3.8 to the sequence $\left(H_{n}\left(G_{t}\right)_{n \in \mathbb{N}^{*}}\right.$.

\section{Simulation techniques}

Two effective methods will be given here in order to construct simulated paths of a stationary non-Gaussian process. A common ingredient of these two methods is the simulation of a particular stationary Gaussian process for which two methods have been examined: the spectral method [4,18,25] and the Markovian model method $[2,11,12]$. As it will be shown later, the choice of the method has some incidence on the method efficiency.

The first stage is to generate the stationary Gaussian process $\left(G_{t}, t \in \mathbb{R}^{+}\right)$with marginal distribution $\mathscr{N}(0,1)$ and autocorrelation function $R_{G}$. The second stage is to generate the random process $\left(Y_{t}^{M}, t \in \mathbb{R}^{+}\right)$given by $Y_{t}^{M}$ $\sum_{n=1}^{M} f_{n} H_{n}\left(G_{t}\right)$ ( $M$ is fixed a priori), where the coefficients $\left(f_{n}\right)$ are obtained either by numerical integration (9) or by Monte-Carlo simulation (14).

\subsection{Determination of $R_{G}$}

The goal is to find a nonnegative definite function $R_{G}$ which minimizes the quantity

$\left\|R(t) \quad R_{M}(t)\right\|_{L^{2}(\mathbb{R}, \mathrm{d} x)} \quad\left\|R(t) \quad \sum_{n=1}^{M}(n !) f_{n}^{2}\left(R_{G}(t)\right)^{n}\right\|_{L^{2}(\mathbb{R}, \mathrm{d} x)}$.

The constraint of nonnegative definite property for the autocorrelation function is rather tricky to include numerically in the minimization algorithm. It can be replaced by a simpler constraint by introducing the spectral density using Bochner theorem. Actually, denoting $S_{G}$ the spectral density function of $\left(G_{t}, t \in R^{+}\right)$(assuming the density exists), the problem becomes:

Minimize the quantity

$\left\|R(t) \quad R_{M}(t)\right\|_{L^{2}(\mathbb{R}, \mathrm{d} x)}$

$$
\left\|R(t) \quad \sum_{n=1}^{M}(n !) f_{n}^{2}\left(\int_{\mathbb{R}} S_{G}(\omega) \mathrm{e}^{\mathrm{i} \omega t} \mathrm{~d} \omega\right)^{n}\right\|_{L^{2}(\mathbb{R}, \mathrm{d} x)},
$$

under the following constraints:

(i) $S_{G}$ nonnegative, (ii) $S_{G}$ even,

(iii) $\int_{\mathbb{R}} S_{G}(\omega) \mathrm{d} \omega \quad 1$.

\subsection{Autocorrelation function determination for the spectral method}

The minimization is here achieved by discretizing in a first step each integral, and using then a global stochastic recursive approximation algorithm (see Ref. [6]):

$\min _{\sigma_{k} \geq 0} \sum_{l}\left(R\left(t_{l}\right) \quad \sum_{n=1}^{M}(n !) f_{n}^{2}\left(\Delta_{\omega} \sum_{k} \sigma_{k} \mathrm{e}^{\mathrm{i} \omega_{k} t_{l}}\right)^{n}\right)^{2}$.

The minimization solution $\left(\sigma_{k}\right)_{k}$ is obtained using for instance a simulated annealing algorithm or any other method as genetic algorithm.

The spectral density $S_{G}$ is then approached by the step function:

$S_{G}(\omega) \quad \sum_{k} \sigma_{k} \rrbracket_{\left[\omega_{k}, \omega_{k+1}\right]}(\omega)$.

The spectral method is used to simulate the stationary Gaussian process $\left(G_{t}, t \in \mathbb{R}^{+}\right)$. Finally the process $\left(Y_{t}^{M}\right.$ $\left.\sum_{n=1}^{M} f_{n} H_{n}\left(G_{t}\right)\right)$ is simulated.

\subsection{Autocorrelation function determination for the Markovian model representation}

The advantage of using a Markovian model is that it yields a much smaller dimension minimization problem than the former method. It is based on the following assumption:

Suppose that $\int_{\mathbb{R}}\left(\ln \left(S_{G}(\omega)\right) /\left(1+\omega^{2}\right)\right) \mathrm{d} \omega>\quad \infty$. Then it implies that there exists $H \in H^{+}(\mathbb{C})$ (Hardy space) such that $[2,12]$

$S_{G}(\omega) \quad|H(i \omega)|^{2}$.

The function $H(\mathrm{i} \omega)[2,12,17,24]$ is either a rational function itself or can be approached by a rational function:

$\frac{\Phi(\mathrm{i} \omega)}{\Psi(\mathrm{i} \omega)}$,

where

(i) $\Phi, \Psi$ are real coefficient polynomials ( $\Psi$ is unitary),

(ii) $\operatorname{deg} \Phi<\operatorname{deg} \Psi$,

(iii) the roots of $\Psi$ lie in $\{\operatorname{Re}(z)<0\}$.

The goal is to minimize the quantity

$\left\|R(t) \quad \sum_{n=1}^{M}(n !) f_{n}^{2}\left(\int_{\mathbb{R}}\left|\frac{\Phi(\mathrm{i} \omega)}{\Psi(\mathrm{i} \omega)}\right|^{2} \mathrm{e}^{\mathrm{i} \omega t} \mathrm{~d} \omega\right)^{n}\right\|_{L^{2}(\mathbb{R}, \mathrm{d} x)}$,

relatively to the coefficients $\left(\phi_{k}\right)_{k=0}^{\operatorname{deg} \Phi}$ and $\left(\Psi_{k}\right)_{k=0}^{\operatorname{deg} \Psi}$ of polynomials $\Phi$ and $\Psi$, respectively, under the constraint that $\Phi$ and $\Psi$ are described as above. 
Remark 4.1. The dimension of this new minimization problem is equal to the number of coefficients of $\Phi$ and $\Psi$ while the dimension of the former minimization problem is equal to the number of points used for calculating the integrals in Eq. (50).

Once the polynomials $\Phi$ and $\Psi$ are determined, it remains to simulate the underlying stationary Gaussian process by the following method.

Let $\left(\xi_{t}, t \in \mathbb{R}^{+}\right)$a random process with values in $\mathbb{R}^{\operatorname{deg} \Psi}$, which is solution of the Itô stochastic differential equation

$\mathrm{d} \xi_{t} \quad A \xi_{t} \mathrm{~d} t \quad \mathrm{~d} Z_{t}$,

where $A$ is the companion matrix of polynomial $\Psi$, and $\mathrm{d} Z_{t} \quad\left(0, \ldots, 0, \mathrm{~d} W_{t}\right)^{\mathrm{T}}$, and $W_{t}$ is the standard Wiener process.

Various schemes exist in order to construct its solution $\left(\xi(0) \quad \xi_{0} ; \xi_{t}, t \in \mathbb{R}^{+}\right)[1,14,26]$.

Let $\left(G_{t}, t \in \mathbb{R}^{+}\right)$be a scalar process defined by

$G_{t} B \xi_{t}$,

where

$B \quad\left(\phi_{0}, \phi_{1}, \ldots, \phi_{\operatorname{deg} \Phi}, 0, \ldots, 0\right) \in \mathbb{R}^{\operatorname{deg} \Psi}$.

Then $\left(G_{t}, t \in \mathbb{R}\right)$ is a Gaussian process which spectral measure has a density given by $|\Phi(\mathrm{i} \omega) / \Psi(\mathrm{i} \omega)|^{2}$.

As in the previous method, the simulation of the nonGaussian process $Y_{t}$ is achieved by constructing the approaching truncated sum

$Y_{t}^{M} \quad \sum_{n=1}^{M} f_{n} H_{n}\left(G_{t}\right)$.

\section{Examples}

As the aim of this paper is to prove the theoretical validity of the proposed simulation methods, we give here just some illustrations of these methods without qualitative comments. Comparison between existing simulation methods of nonGaussian processes will be the object of a future work.

\subsection{Data}

(i) Five statistical moments are given

$$
\begin{array}{llllllll}
\mu_{1} & 0, & \mu_{2} & 1, & \mu_{3} & 2, & \mu_{4} & 9, \\
\mu_{5} & 44,
\end{array}
$$

(ii) the spectral density is given by

$S(\omega) \quad \frac{1}{2 \pi} \frac{100}{270} \frac{1+0.6558 \omega^{2}}{\left(1+0.2459 \omega^{2}\right)^{11 / 6}}$.

(iii) $M \quad$ 4, 1024 discretization points are used and 1000 simulations are performed.
Using the ergodic property of process $Y_{t}$, the various statistics are estimated using each point of simulated trajectories.

\subsection{Case where the marginal distribution is given}

We consider the case where the one-dimensional marginal distribution of the non-Gaussian process is given. Let $X$ a random variable with the exponential distribution $\exp (1)$ and let $Y \quad X \quad 1$, then

$$
\begin{aligned}
& \mathbb{E}(Y) \quad 0, \quad \mathbb{E}\left(Y^{2}\right) \quad 1, \quad \mathbb{E}\left(Y^{3}\right) \quad 2, \quad \mathbb{E}\left(Y^{4}\right) \quad 9, \\
& \mathbb{E}\left(Y^{5}\right) \quad 44 .
\end{aligned}
$$

The inverse of the cumulative distribution function of $Y$ is obtained easily:

$F_{Y}{ }^{1}(y) \quad 1 \quad \ln (1 \quad y)$.

The cumulative distribution function of the random variable

$F_{Y}{ }^{1} \circ F_{G}(G) \quad 1 \quad \ln \left(1 \quad F_{G}(G)\right)$

is $F_{Y}$. The coefficients $f_{n}$ can be obtained by a numerical integration:

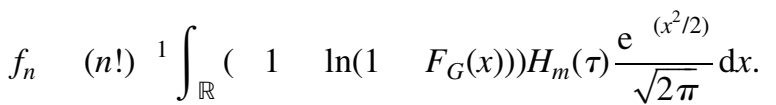

\subsubsection{Simulation using the spectral approach}

The comparison between the target and the estimated spectral density is shown on Fig. 1. The histogram of $Y_{t}$ marginal distribution is compared to the target marginal distribution, Fig. 2. The comparison between the target and the estimated statistical moments is given in Table 1 .

\subsubsection{Simulation using the Markovian model}

For this application $\Phi$ and $\Psi$ are defined by:

$\Phi(x) \quad\left(\alpha_{1}+\beta_{1} x\right)\left(\alpha_{2}+\beta_{2} x\right)$,

$\Psi(x) \quad\left(x^{2}+2 \gamma_{1} \delta_{1} x+\delta_{1}^{2}\right)\left(x^{2}+2 \gamma_{2} \delta_{2} x+\delta_{2}^{2}\right)$,

where $\left(\alpha_{1}, \beta_{1}\right),\left(\alpha_{2}, \beta_{2}\right) \in \mathbb{R} \backslash\{(0,0)\}$ and $\gamma_{1}, \gamma_{2}, \delta_{1}, \delta_{2}$ are positive real numbers. As in Section 5.2.1, the same comparisons can be found in Figs. 3 and 4 and Table 2. Even if the Markovian model brings another level of approximation, since the power spectral density is approached by a rational function, the two approaches give a very good estimate of the target power spectral density.

Table 1

Target and estimated statistical moments, marginal distribution given/spec tral method

\begin{tabular}{lcc}
\hline Moment & Target & Estimated \\
\hline Order 2 & 1.00 & 1.00 \\
Order 3 & 2.00 & 1.976 \\
Order 4 & 9.00 & 8.80 \\
Order 5 & 44.00 & 42.18 \\
\hline
\end{tabular}




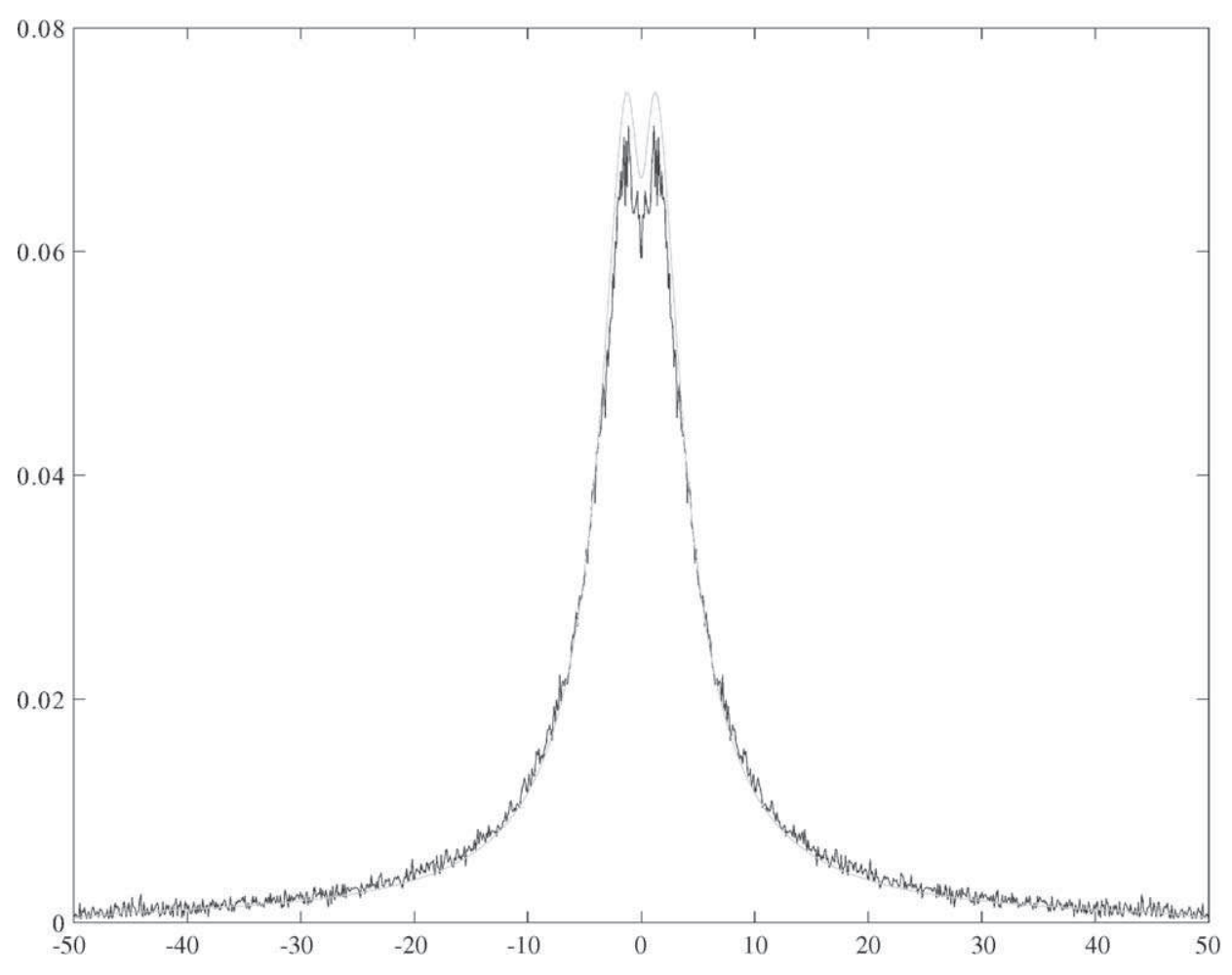

Fig. 1. Comparison between target and estimated spectrum, marginal distribution given/spectral method.

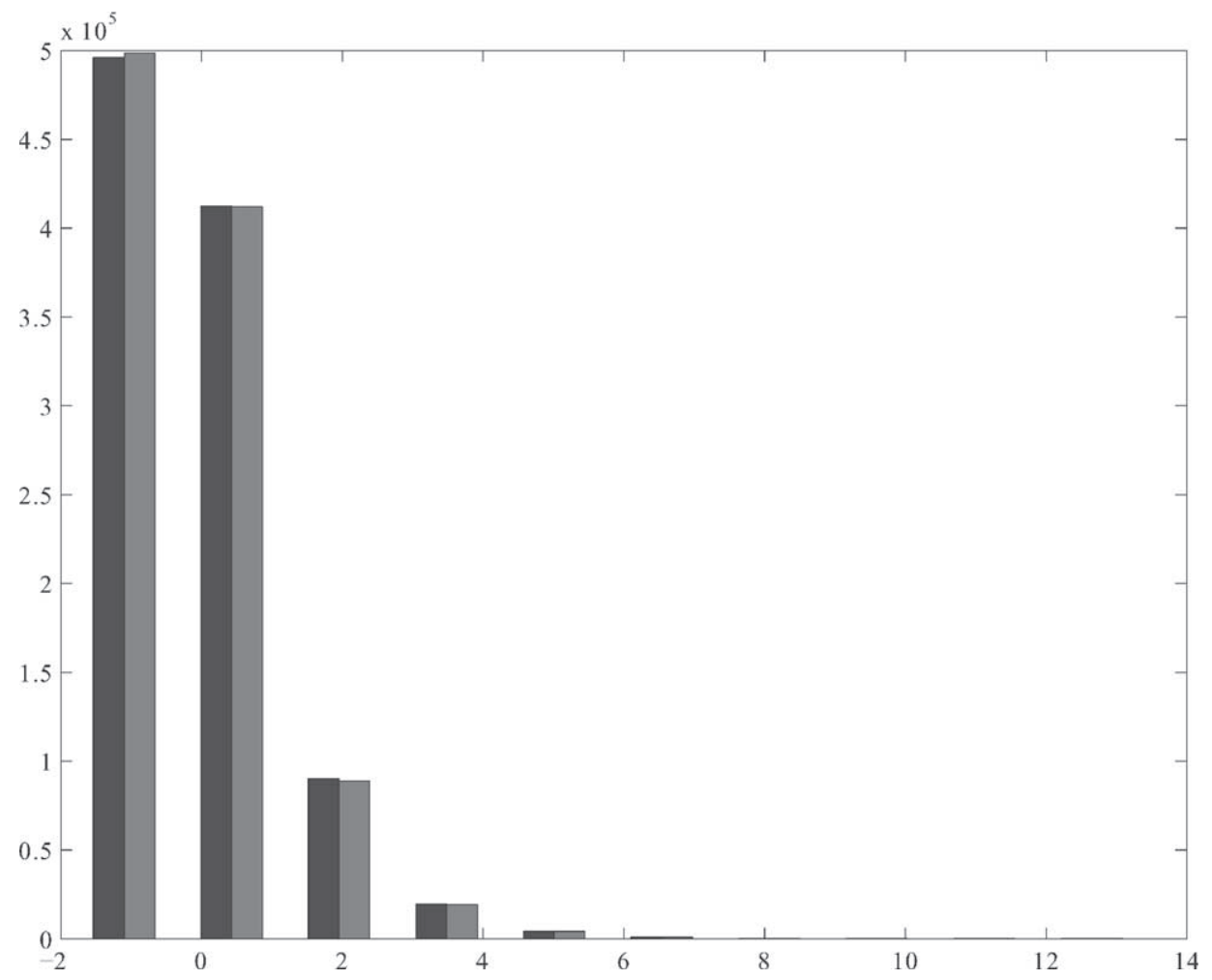

Fig. 2. Histogram, comparison between target and simulated marginal distribution/spectral method. 


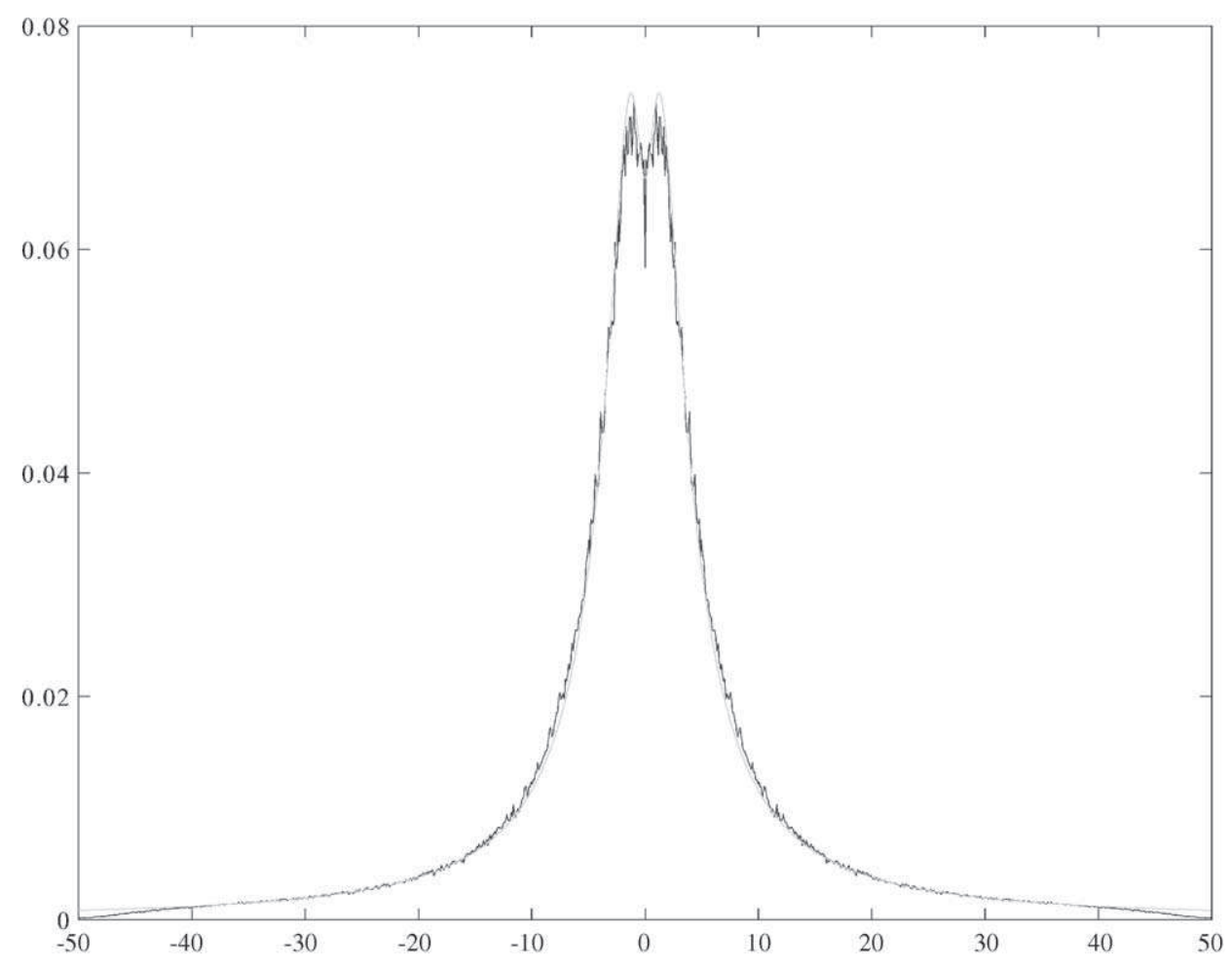

Fig. 3. Comparison between target and estimated spectrum, marginal distribution given/Markovian model.

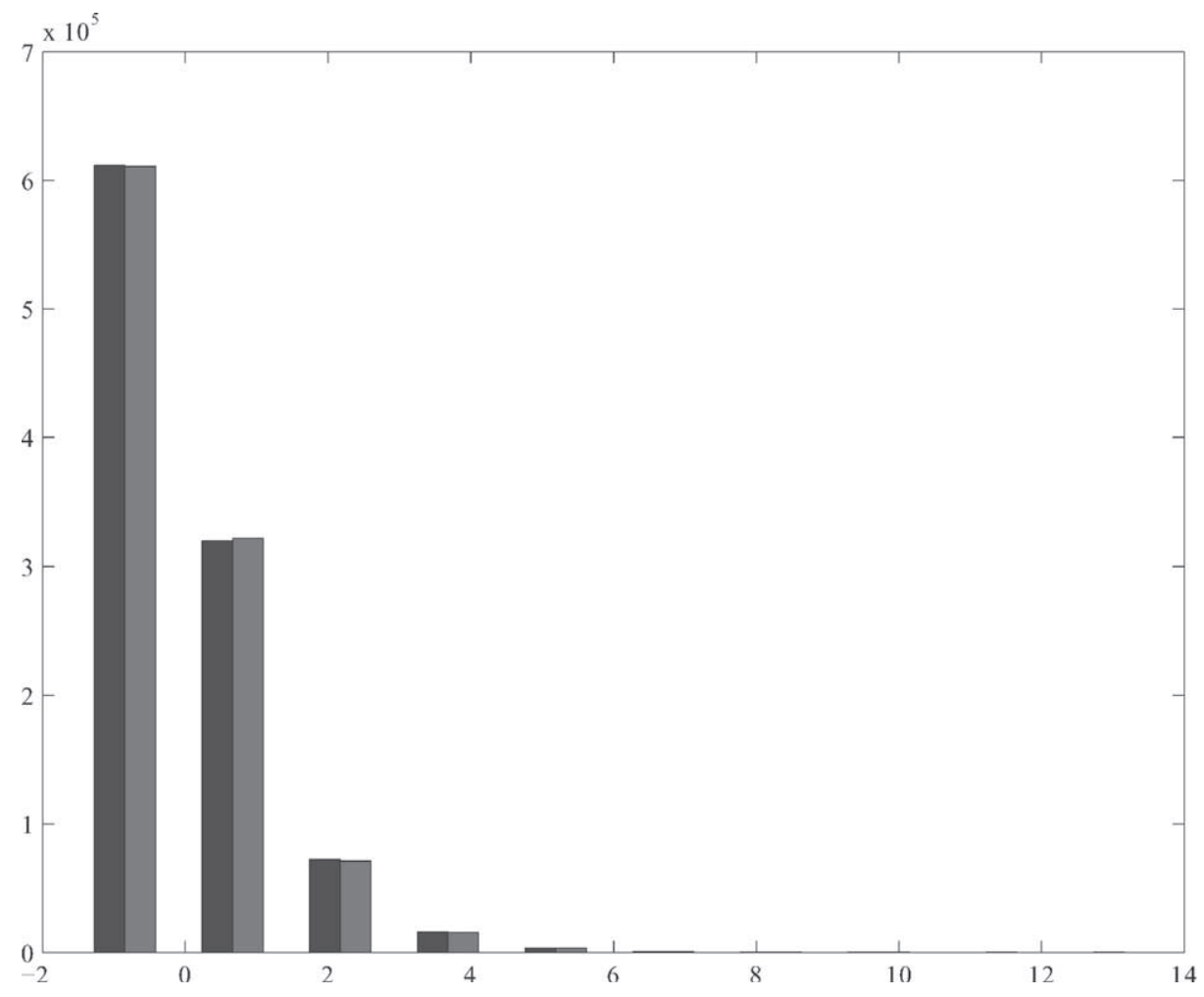

Fig. 4. Histogram, comparison between target and simulated marginal distribution/Markovian model. 
Table 2

Target and estimated statistical moments, marginal distribution given/ Markovian model

\begin{tabular}{lrc}
\hline Moment & Target & Estimated \\
\hline Order 2 & 1.00 & 1.00 \\
Order 3 & 2.00 & 1.99 \\
Order 4 & 9.00 & 9.03 \\
Order 5 & 44.00 & 45.48 \\
\hline
\end{tabular}

\subsection{Case where a finite number of statistical moments are given}

In the case where only a number of statistical moments are given, we have to generate a random variable $Y$ with given moments and which distribution is absolutely continuous with respect to the Lebesgue measure (see Section 2 and Ref. [5]). The inverse of the cumulative distribution function of $Y$ is then constructed numerically. Moreover, contrary to the preceding case, the coefficients $f_{n}$ appearing in the approaching sequence are estimated using MonteCarlo simulations. The results concerning the spectral density function are shown in Figs. 5 and 6 for, respectively, the spectral and Markovian approach. Results concerning moments are, in the same way, resumed in Tables 3 and 4. As in the preceding case, the agreement is excellent between estimated and target quantities.

Looking at the optimization problem dimension, it is obvious that the Markovian approach needs a lesser computational effort than the spectral approach: eight parameters
Table 3

Target and estimated statistical moments, fixed moments/spectral method

\begin{tabular}{lcc}
\hline Moment & Target & Estimated \\
\hline Order 2 & 1.00 & 1.01 \\
Order 3 & 2.00 & 2.05 \\
Order 4 & 9.00 & 8.90 \\
Order 5 & 44.00 & 41.13 \\
\hline
\end{tabular}

Table 4

Target and estimated statistical moments, fixed moments/Markovian model

\begin{tabular}{lcc}
\hline Moment & Target & Estimated \\
\hline Order 2 & 1.00 & 1.01 \\
Order 3 & 2.00 & 2.03 \\
Order 4 & 9.00 & 8.88 \\
Order 5 & 44.00 & 41.38 \\
\hline
\end{tabular}

to optimize for the first method versus 1024 parameters for the spectral approach. Nevertheless, it appears that the optimization procedure converges very rapidly in this last case for this particular application. This can be explained by the fact that the starting point (the target spectral density) of the optimization procedure is 'close' to the correct Gaussian spectral density. This property was observed by the authors of Ref. [20]. Mathematically, this is explained by the convergence speed of $R_{M}$ towards $R_{Y}$, given by relation (28). Moreover, when only one term is kept in the expansion, $M \quad 1$ :

$\left|R_{Y}(t) \quad R_{G}(t)\right| \leq\left|R_{G}(t)\right|^{2}$

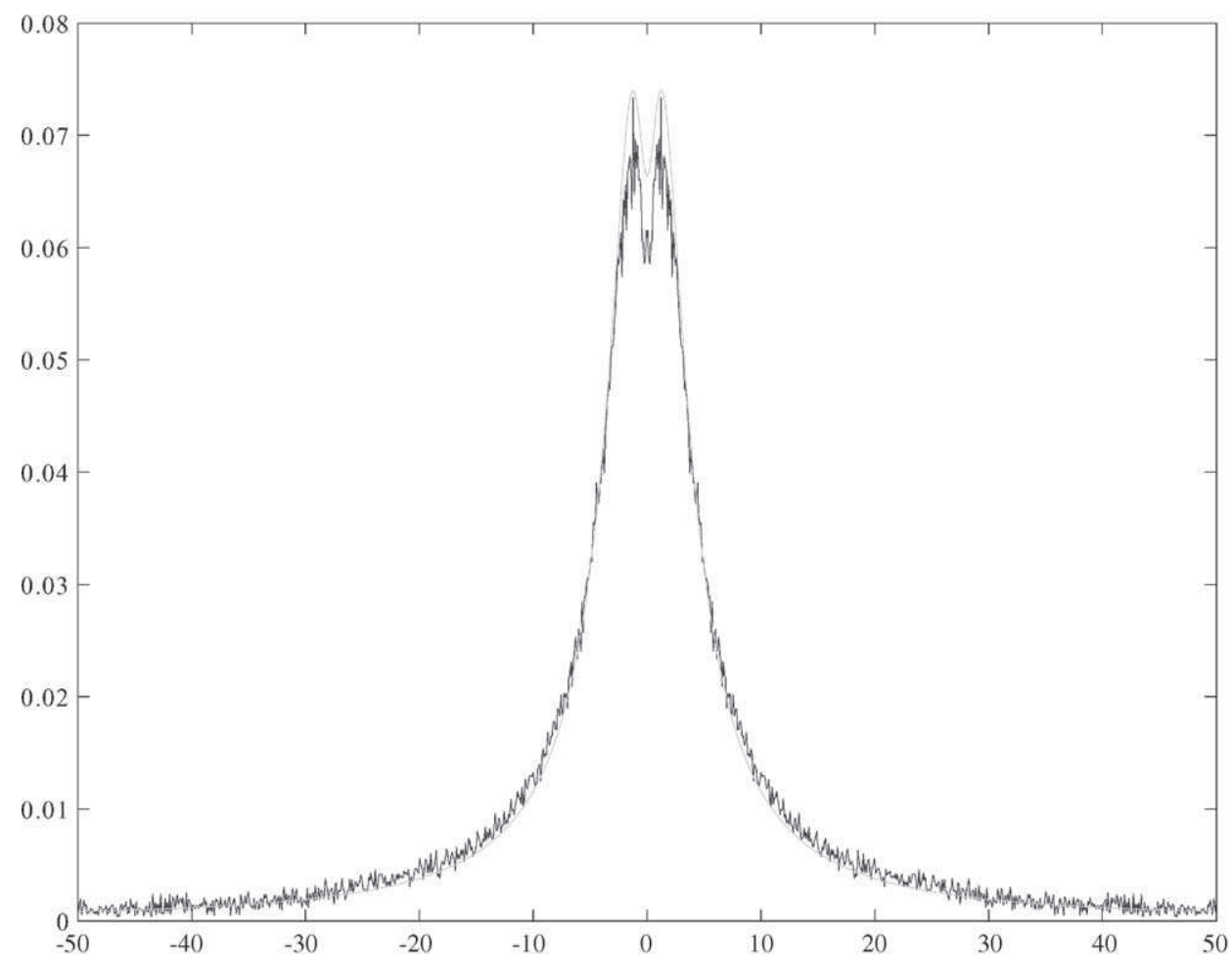

Fig. 5. Comparison between target and estimated spectrum, fixed moments/spectral method. 


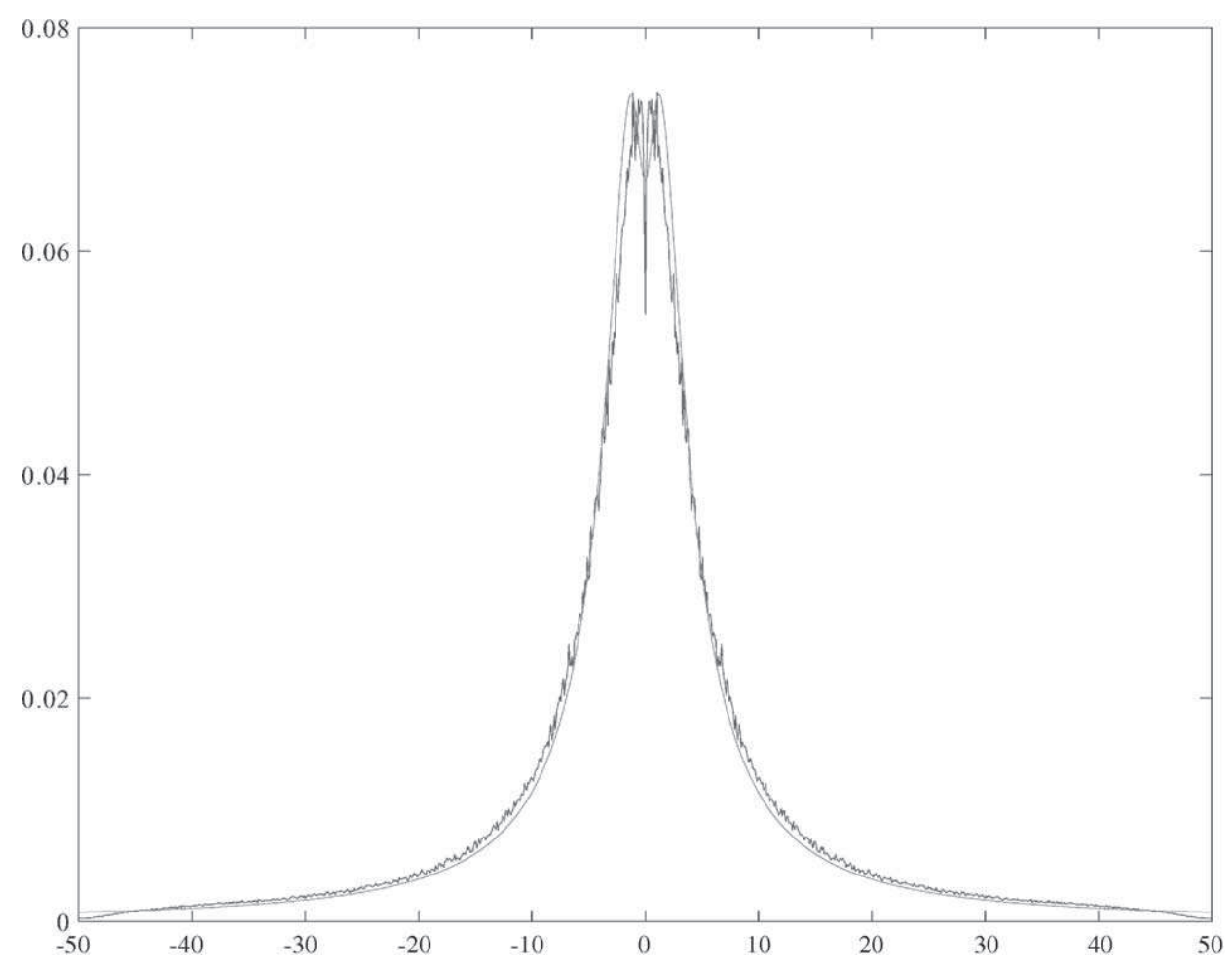

Fig. 6. Comparison between target and estimated spectrum, fixed moments/Markovian model.

with $\left|R_{G}(t)\right| \leq 1$. A last comment considering the optimization problem for the spectral approach is that its overall dimension could be reduced in a significant manner if other numerical integration methods were used, such as the Gauss point method. And this should be used for random fields $Y(t), t \in \mathbb{R}^{d}$.

\subsection{Case of a scalar homogeneous random field}

\subsubsection{Data}

(i) The same statistical data as in the previous example are utilized.

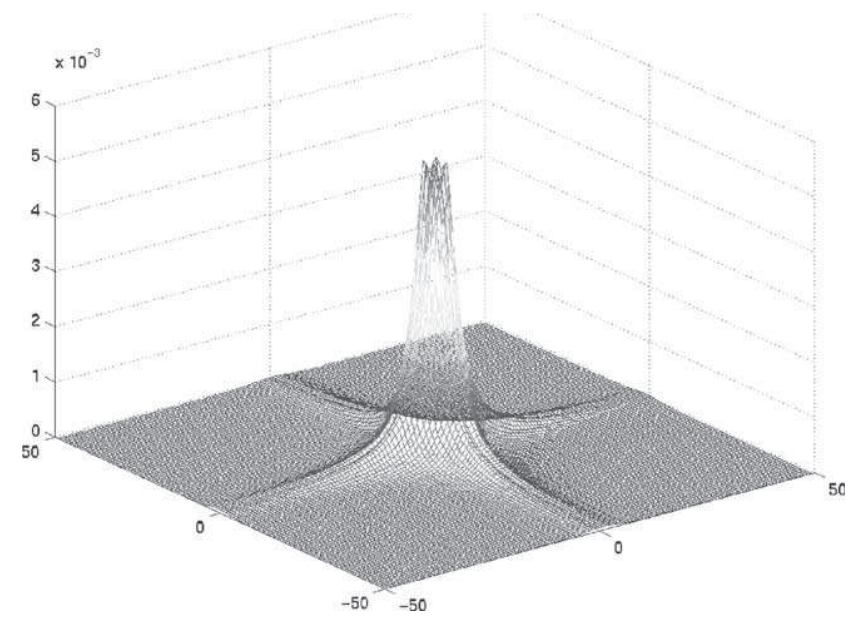

Fig. 7. Target spectrum, random field case. (ii) The spectral density is given by

$$
\begin{aligned}
S\left(\omega_{1}, \omega_{2}\right) \quad & \frac{1}{2 \pi} \frac{100}{270} \frac{1+0.6558 \omega_{1}^{2}}{\left(1+0.2459 \omega_{1}^{2}\right)^{11 / 6}} \\
& \times \frac{1}{2 \pi} \frac{100}{270} \frac{1+0.6558 \omega_{2}^{2}}{\left(1+0.2459 \omega_{2}^{2}\right)^{11 / 6}} .
\end{aligned}
$$

(iii) $M \quad 4,128 \times 128$ discretization points are utilized and 1000 simulations are performed.

The simulation of the underlying Gaussian random field is performed here using the spectral method. Although one

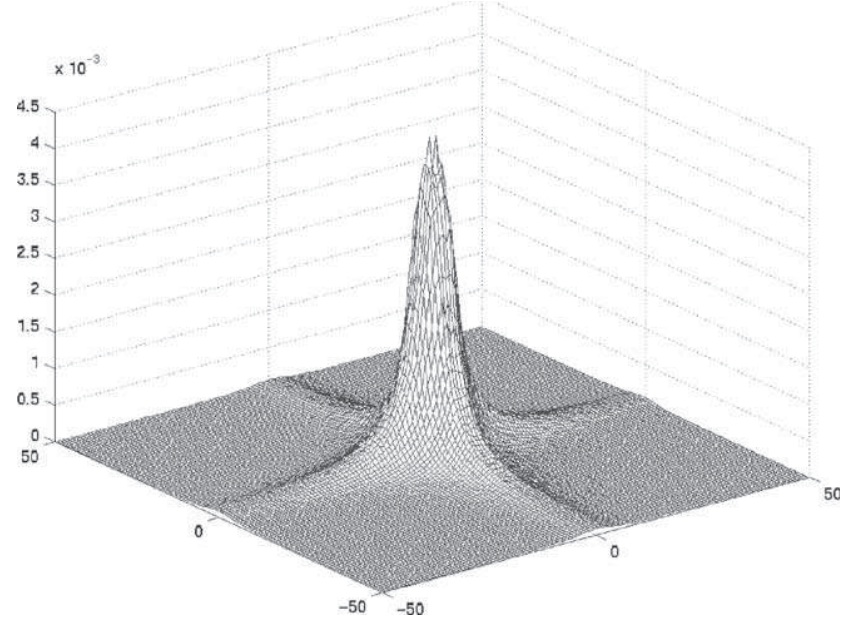

Fig. 8. Estimated spectrum, marginal distribution given. 


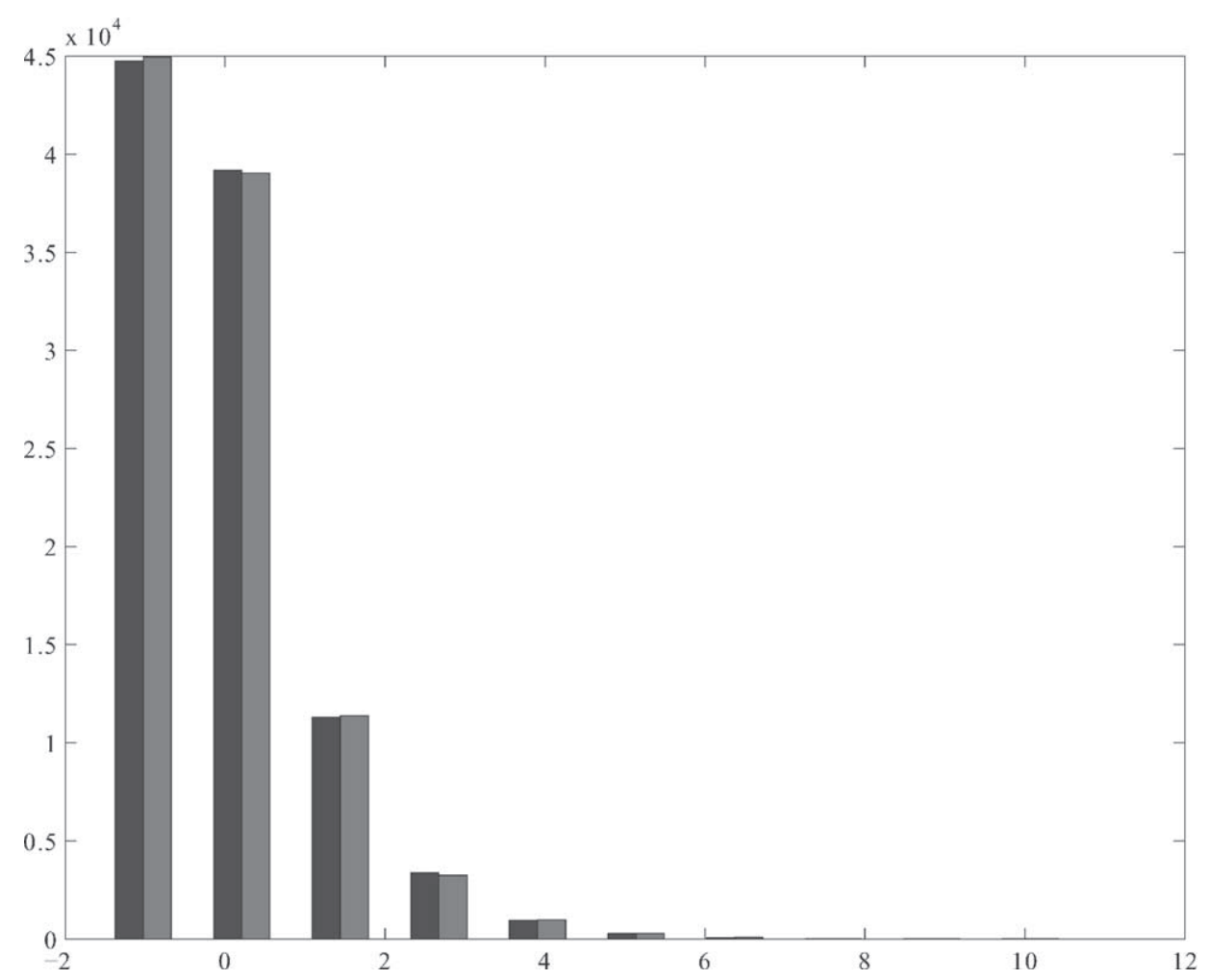

Fig. 9. Histogram, comparison between target and simulated marginal distribution.

can construct a Markovian model for random field yielding a stochastic partial differential equation, it is difficult to integrate it because one has to discretize a partial differential equation on a domain of $\mathbb{R}^{d}$. Therefore the Markovian model is not effective for random fields.

In the case where the marginal distribution is given, the target spectral density is shown in Fig. 7 and has to be compared to the estimated spectral density shown in Fig. 8. Fig. 9 depicts the comparison of the target and estimated marginal distribution histogram. Finally the comparison between the target and the estimated statistical moments is given in Table 5 .

The analogous results of the case 2 data are given in Fig. 10 and Table 6.

Here again, the agreement between the estimated and the target quantities is very good.

\section{Conclusion}

In this paper, various convergence results for Hermite

Table 5

Target and estimated statistical moments, marginal distribution given/spec tral method

\begin{tabular}{lcc}
\hline Moment & Target & Estimated \\
\hline Order 2 & 1.00 & 1.00 \\
Order 3 & 2.00 & 2.00 \\
Order 4 & 9.00 & 9.07 \\
Order 5 & 44.00 & 44.53 \\
\hline
\end{tabular}

polynomial expansion of a Gaussian process are given. These results act as lifeguards for simulation techniques based on such Hermite polynomial expansions. In particular, it can be proved under some regularity assumption that the speed of convergence of Hermite expansion correlation function towards the non-Gaussian correlation function is controlled by the quantity $(M \times M)^{1}$ where $M$ is the number of polynomials in the sum. Various algorithms are also given, allowing to construct simulations of general nonGaussian processes. The simulation method relies on the simulation of a Gaussian process, which can be simulated

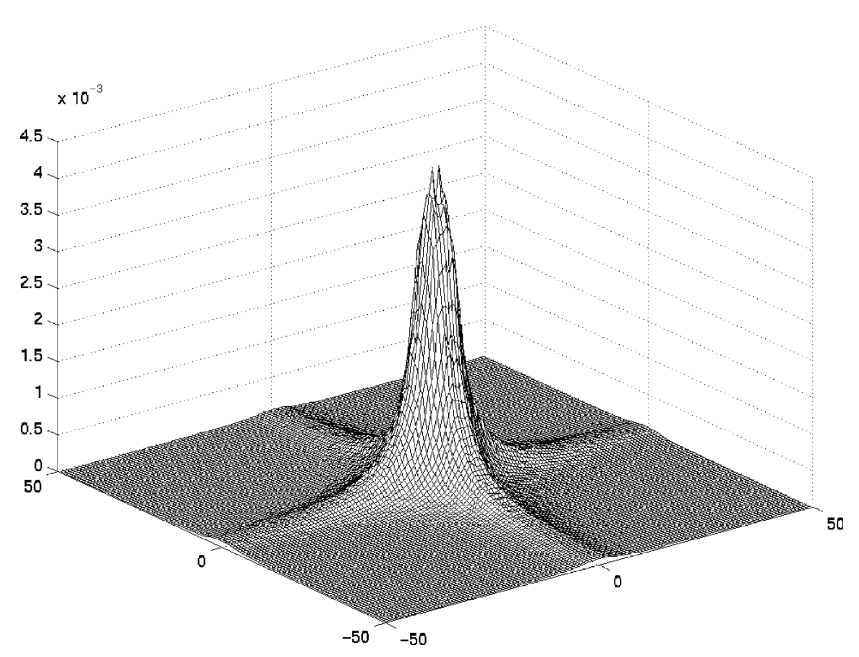

Fig. 10. Estimated spectrum, fixed moments. 
Table 6

Target and estimated statistical moments, fixed moments/spectral method

\begin{tabular}{lcc}
\hline Moment & Target & Estimated \\
\hline Order 2 & 1.00 & 1.00 \\
Order 3 & 2.00 & 2.01 \\
Order 4 & 9.00 & 9.17 \\
Order 5 & 44.00 & 45.58 \\
\hline
\end{tabular}

using either a spectral approach, or a Markovian approach. This last method yields a lesser computational effort, but which is not effective for random fields. Various examples illustrate the soundness of the method. The general method can be extended in theory to non-stationary random processes, but, in practice, such a generalization would lead to numerical and estimation difficulties, the first one being to deal with time dependent statistical characteristics of the non-Gaussian process and time (or space) dependent estimations of real-life random phenomena. Extension to vector valued random process is also straightforward, since it is based on the simulation of a vector valued Gaussian process and on the simulation of a vector valued random variable.

\section{References}

[1] Bernard P, Fleury G. Stochastic Newmark method. Spanos PD, editor. Comput Stochast Mech 1999:365 73.

[2] Bernard P, Fogli M, Bressolette P, Lemaire M. Un algorithme de simulation stochastique par Markovianisation approchée application à la mécanique aléatoire. J Mécanique Théorique Appliquée 1984;3(6):905 50.

[3] Declercq D. Apport des polynômes d'Hermite à la modélisation non Gaussienne et tests statistiques associés. Thesis, Cergy Pontoise University; 1998.

[4] Deodatis G, Shinozuka M. Simulation of stochastic processes by spectral representation. Appl Mech Rev 1991;44(4):191 204.

[5] Devroye L. Non uniform random variate generation. Berlin: Springer, 1986.

[6] Duflo M. Random iterative models. Berlin: Springer, 1997.

[7] Gioffrè M, Giusella V, Grigoriu M. Simulation of non Gaussian field applied to wind pressure fluctuations. Probab Engng Mech 2000;15:339 45 .
[8] Grigoriu M. Simulation of stationary non Gaussian translation processes. J Engng Mech ASCE 1998;124(2):121 7.

[9] Gurley KR, Kareem A. Simulation of non Gaussian processes. Spanos PD, editor. Comput Stochast Mech 1999:11 20.

[10] IASSAR. A state of the art report on computational stochastic mechanics. Shinozuka M, Spanos PD, editors. Probab Engng Mech 1997; 12:197 321 .

[11] Krée P, Soize C. Markovianization of non linear oscillators with colored input. Actes de la conférence de mécanique aléatoire à l'École Polytechnique de Turin; 1982. p. 13550.

[12] Krée P, Soize C. Mathematics of random phenomena. Dordrecht: Reidel, 1986.

[13] Loève M. Probability theory. 2nd ed. Princeton: Van Nostrand, 1960.

[14] Pardoux E, Talay D. Discretization and simulation of stochastic differential equations. Acta Appl Math 1985;3:23 47.

[15] Parzen E. Stochastic processes. San Francisco, CA: Holden Day, 1962.

[16] Poirion F. Numerical simulation of homogeneous non Gaussian random vector fields. J Sound Vibrat 1993;160(1):25 42.

[17] Poirion F. Effective methods in stochastic non linear dynamics. Aero servoelasticity applications. In: Bouc R, Soize C, editors. Progress in stochastic structural dynamics. Paris: CNRS, 1999.

[18] Poirion F, Soize C. Numerical methods and mathematical aspects for simulation of homogeneous and non homogeneous Gaussian fields. In: Krée P, Wedig W, editors. Probabilistic Methods in Applied Physics, Lecture Notes in Physics 451. Berlin: Springer, 1995. p. 1753.

[19] Popescu R, Deodatis G, Prevost JH. Simulation of homogeneous non Gaussian stochastic vector fields. Probab Engng Mech 1998;13(1):1 13.

[20] Sakamoto S, Ghanem R. Simulation of non Gaussian fields with the Karhunen Loeve and polynomial chaos expansions. Engineering Mechanics Conference, Baltimore (USA); June 1999.

[21] Sakamoto S, Ghanem R. Simulation of multi dimensinal non Gaussian non stationary random fields. Probab Engng Mech 2002 in press.

[22] Shinozuka M. Simulation of multivariate and multidimensional random processes. J Acoust Soc Am 1971;49(1):357 67.

[23] Shohat J, Tamarkin J. The problem of moments, Mathematical Surveys. Providence, RI: American Mathematical Society, 1963 Number 1.

[24] Soize C. Méthodes mathématiques en analyse du signal. Paris: Masson, 1993.

[25] Spanos PD, Zeldin BA. Monte Carlo treatment of random fields: a broad perspective. Appl Mech Rev 1998;51(3):219 37.

[26] Talay D. Simulation and numerical analysis of stochastic differential systems: a review. Technical Report 1313, INRIA; 1990.

[27] Yamazaki F, Shinozuka M. Digital generation of non gaussian stochastic fields. J Engng Mech 1988;114(7):1183 97. 